\title{
A Fate Worse Than Death? The Well-Being of Patients Diagnosed as Vegetative With Covert Awareness
}

\author{
Mackenzie Graham ${ }^{1}$ (DD
}

Accepted: 12 September 2017 / Published online: 23 October 2017

(C) The Author(s) 2017. This article is an open access publication

\begin{abstract}
Patients in the vegetative state are wholly unaware of themselves, or their surroundings. However, a minority of patients diagnosed as vegetative are actually aware. What is the well-being of these patients? How are their lives going, for them? It has been argued that on a reasonable conception of well-being, these patients are faring so poorly that it may be in their best interests not to continue existing. I argue against this claim. Standard conceptions of wellbeing do not clearly support the conclusion that these patients would be better off having lifesustaining treatment withdrawn, and in fact, it may be possible for these patients to retain a passable level of well-being. I suggest that further research into the subjective experiences of these patients will allow us to better promote their well-being.
\end{abstract}

Keywords Well-being $\cdot$ vegetative state $\cdot$ disorders of consciousness $\cdot$ neuroimaging $\cdot$ prudential interests

\section{Introduction}

The vegetative state is a neurological condition in which an individual is awake, but wholly unaware of themselves, or their environment (Multi-Society Task Force 1994). Individuals in the vegetative state show no evidence of purposeful or voluntary behavioural responses to stimuli, and no evidence of attention, intention, or learned responses. Occasional movements are merely reflexive, and although they may sometimes appear to smile or cry, and occasionally utter grunts or moans, they show no evidence of language comprehension or capacity for expression (Multi-Society Task Force 1994). This condition may be transient, marking a stage in recovery from acute or chronic brain damage, or more long-term. According to the Royal College of Physicians, the vegetative state is considered 'persistent' after approximately 4 weeks. Conversely, the chances of regaining consciousness 3 months after non-traumatic

Mackenzie Graham mgraha44@uwo.ca 
brain damage, or 12 months after traumatic brain injury, are extremely low, at which time the vegetative state is considered 'permanent.' (Multi-Society Task Force 1994; Royal College of Physicians 2003). In this paper, I focus on those patients diagnosed as being in either a 'persistent' or 'permanent' vegetative state (and simply use 'vegetative state' to refer to both).

Neuroscientific research over the last decade has demonstrated that a minority of patients (19\%) diagnosed as vegetative are actually 'covertly aware' (Owen et al. 2006; Monti et al. 2010; Bardin et al. 2012; Naci et al. 2013). These patients, who repeatedly satisfy all of the behavioural criteria for a diagnosis of vegetative at the bedside, are capable of volitionally modulating their brain activity, in response to the instructions of researchers. In the classic paradigm, patients are asked to imagine performing 'mental imagery', such as imagining hitting a tennis ball, or navigating the rooms in their home, in order to produce a characteristic brain response. Using functional magnetic resonance imaging (fMRI), researchers can detect activity in the areas of the brain which correspond to task performance, thus providing robust evidence that the patient purposefully engaged in a specific brain activity, and is consciously aware (Monti et al. 2010). ${ }^{1}$ Stender et al. (2014) have also used 18-fluorodeoxyglucose positron emission tomography (FDG-PET) to detect brain activation patterns consistent with minimal consciousness in patients diagnosed as vegetative.

The discovery of covert awareness in these patients is significant, because it allows for the possibility that they have subjective interests, which may promote or detract from their wellbeing. For example, these patients may be capable of suffering as a result of physical pain, or experiencing enjoyment as a result of physical pleasure (Graham et al. 2015). When we think about what contributes to our well-being, we do so from a subjective point of view; whether something is good or bad for me. Awareness seems to be an important part of what allows us to assume this subjective point of view, according to which something can be good or bad, for us.

How are these individuals faring? I would suggest that our pre-reflective intuitions are that these individuals are faring rather poorly indeed. Whatever we think that the constituents of well-being are, these individuals are probably lacking them. This intuition is exemplified in the work of Kahane and Savulescu (2009). These authors adopt a pluralistic, 'common-sense' account of well-being, which draws on hedonistic, desire-satisfaction, and objective list theories. Given this common-sense view, they suggest, patients diagnosed as vegetative with covert awareness are likely faring so poorly, that others may have a moral obligation to withdraw them from life-sustaining treatment.

Against this intuition, I will argue that individuals diagnosed as vegetative with covert awareness are not faring so poorly that it would be in their best interests to stop existing. We currently know very little about what life is like for these individuals, about how they experience the world. Therefore, applying a 'common-sense' notion of well-being of the sort that Kahane and Savulescu are advocating requires that we make various assumptions about these individuals, their experiences, and what is 'good for' them. And, while rather pessimistic

\footnotetext{
${ }^{1}$ Patients are diagnosed as vegetative on the basis of a behavioural assessment; the lack of a behavioural response to stimuli at the bedside results in the diagnosis of vegetative. Thus, patients who demonstrate covert awareness using mental imagery, or other neural responses, still satisfy the behavioural definition of the vegetative state. For this reason, it has been suggested that functional neuroimaging data be incorporated into the diagnostic criteria for vegetative state, in concert with behavioural diagnostic tools (Coleman et al. 2009; Laureys and Schiff 2012; Gosseries et al. 2014; Peterson et al. 2015). However, these techniques remain investigational in this patient population, and changes to the diagnostic criteria of the vegetative state have yet to gain wide acceptance. Several names have been proposed for patients who fit the behavioural diagnosis of vegetative, but whose demonstration of covert awareness differentiates them from 'classic' cases, in which awareness of any kind is presumed to be absent. In this paper, I refer to these patients as 'patients diagnosed as vegetative with covert awareness.'
} 
assumptions may support our pre-reflective intuitions regarding the well-being of these individuals, I am not convinced that these assumptions are justified. In what follows, I show that the 'common-sense' notion of well-being Kahane and Savulescu adopt does not support their pessimistic conclusion. While these individuals may never reach the level of well-being they would have if they had not become vegetative with covert awareness, they may still achieve a level of well-being sufficient to justify maintaining their lives. We should therefore be open to revising our intuitions about the well-being of these patients, and focus our efforts on promoting their well-being.

In their paper, Kahane and Savulescu question why the discovery of consciousness in patients previously diagnosed as vegetative is morally significant. They conclude that consciousness — specifically, 'phenomenal consciousness' - does not itself make an individual's life worth living. For Kahane and Savulescu, "a state is phenomenally conscious if it has a phenomenal character, if there is something "it is like" to be in that state" (Kahane and Savulescu 2009). Conversely, they use the term 'sapience' to encompass complex cognitive and motivational states and capacities, a "rich mentality" that supports mental states like beliefs, desires, and capacities like rationality. A 'significant' degree of sapience is required to achieve other kinds of goods which arguably contribute to well-being, such as knowledge, achievement, and friendship. Thus, the presence of consciousness in patients diagnosed as vegetative with covert awareness "does not by itself show that it is in the patient's best interests to go on living" (Kahane and Savulescu 2009). In this respect, I agree with these authors. Whether it is best for such a patient to go on living depends on to what extent their well-being interests are satisfied, or frustrated. However, I disagree with Kahane and Savulescu that consideration of the wellbeing of these patients points towards withdrawing them from life-sustaining treatment.

One of the challenges of discussing patients diagnosed as vegetative with covert awareness is the heterogeneity of this population, with respect to their residual cognitive capacities. On the one hand, each of these patients will have been diagnosed as being in a vegetative state based on a behavioural examination at the bedside; they will have failed to produce any voluntary behavioural response to stimuli. They will also have demonstrated their 'covert awareness' through successful completion of some neuroimaging-based task. This shows that these individuals retain language comprehension, short-term memory, and the ability to focus their attention (Monti et al. 2010).

On the other hand, research interventions have demonstrated that some patients diagnosed as vegetative with covert awareness retain more sophisticated cognitive capacities like executive function (cognitive processes involved in reasoning, planning, and problem solving) (Naci et al. 2014), and the ability to experience humor (Fiacconi and Owen 2016), which may imply the preservation of other cognitive capacities. Moreover, some patients diagnosed as vegetative with covert awareness can functionally communicate with researchers via neuroimaging, by answering yes-or-no questions. However, not all patients who demonstrate covert awareness also demonstrate these other cognitive capacities, and of those that do, it is not clear what further cognitive capacities they retain. It is possible, perhaps even likely, that the cognitive capacities of some individuals diagnosed as vegetative with covert awareness may be much greater than others. For this reason, organizing these patients as either 'conscious with minimal sapience', or 'conscious with significant sapience', as Kahane and Savulescu do, is an oversimplification. Without further investigation of their residual cognitive capacities, it is unclear whether a patient diagnosed as vegetative with covert awareness retains 'minimal' or 'significant' sapience, or what aspects of sapience they retain, and to what degree. In what follows, I will specify when I am referring to those individuals with more preserved cognitive 
function (i.e., 'significant sapience'), as opposed to any individual diagnosed as vegetative with covert awareness.

\section{Theories of Well-Being}

The philosophical study of well-being is the study of what is non-instrumentally good for a person. A theory of well-being is concerned with providing an account of prudential value, of what makes one's life (or a part of one's life) go better from the point of view of the individual whose life it is.

A necessary feature of any theory of well-being is its descriptive adequacy; how well the theory captures our beliefs and attitudes about well-being. For most theories, this amounts to capturing our considered intuitions about what it means to fare well as a healthy, adult human being. However, it is not obvious that the nature of well-being is the same for all subjects of well-being. For example, what it means for a child to be faring well is different than what it means for an adult to be faring well (Skelton 2016). For a young child, welfare may consist of experiencing pleasure and avoiding pain, whereas for an adult, welfare may consist of something more complex (e.g., that their desires are satisfied, or that they authentically endorse the conditions of their life). Accordingly, a theory of well-being for patients diagnosed as vegetative with covert awareness must adequately capture our strongly-held beliefs about what it means to fare well as an individual like this.

Kahane and Savulescu presume that what it means to fare well for these individuals is the same as what it means to fare well for a healthy, adult human, and that patients diagnosed as vegetative with covert awareness simply are not faring well according to this standard. Contrarily, I think that evaluating how these individuals are faring from the perspective of healthy adult humans gives a potentially misleading account of their well-being. Specifically, it overlooks the ways in which the needs, values, and attitudes of these individuals may have changed as a result of their injuries, and yields an overly pessimistic view of their well-being.

\section{Experiential Interests}

Let us begin by considering one of the most basic respects in which our lives can go better or worse for us. According to hedonist accounts of welfare, what is prudentially valuable is experiencing pleasure, and avoiding pain. On such a view, my life is going well, for me, to the extent that my pleasurable experiences are, on balance, greater than my painful experiences. A hedonist view of welfare makes sense as a starting point for patients diagnosed as vegetative with covert awareness. In general, the experience of pleasure makes a life go better, and the experience of pain makes a life go worse; it seems reasonable that these are ingredients of welfare for patients diagnosed as vegetative with covert awareness, just as they are for other subjects of welfare. And, if these individuals have any prudential interests at all, its seems most likely that they would have experiential interests.

The care and symptom management required by individuals diagnosed as vegetative with covert awareness is extensive, in both the acute and chronic phases. Caregivers must monitor for skin injuries and pressure ulcers, as well as articular deformations, muscle spasticity, nutrition, hydration and deglutition problems, respiratory and cardiovascular function, intracranial pressure, as well as the activities of daily living (e.g., hygiene, bathing, feeding, or dressing). Any of these issues might lead to significant discomfort or pain on the part of these 
individuals, thus compromising their experiential interests. However, diligent, attentive care can minimize the experiential harms which accrue to these individuals as a result of pain or discomfort. And, providing them with physical comforts (e.g., massage, passive exercise) and adequate stimulation (e.g., music, audio-books, television) can help to provide a positive balance of experiential interests.

It seems at least possible that on a strictly hedonist account of well-being, patients diagnosed as vegetative with covert awareness might actually fare reasonably well. Depending on the extent of their cognitive limitations, (i.e., if these patients possess minimal sapience) it may be the case that the range of their experience is limited to physical pleasures and pains. If this is the case, having their basic physical needs met (e.g., nutrition, hydration, hygiene, etc.), along with minimal painful experiences, would seem to be sufficient for a moderate level of well-being. While many of the sorts of pleasures which contribute to well-being might be unavailable to them, so to would these individuals be immune from the many pains which can compromise the well-being of healthy individuals. These patients would simply have a highly-circumscribed set of well-being ingredients. Looking from the outside, this does not seem like it would be a particularly enviable life, but from the inside, if the experience of these patients is essentially that of a 'contented infant', we might think that they are not so poorly off, at least given a hedonist conception of well-being.

However, Kahane and Savulescu suggest that even if these individuals did not experience pain, but only highly pleasant states of consciousness, it is not clear that we ought to keep them alive. Indeed, we might question whether maintaining the lives of such patients is the best use of scarce health-care resources; while these individuals may be faring well by a certain standard, they would not stand to lose very much if they did not continue to exist. We ought to devote our resources to where they would have a greater impact. This seems to be Kahane and Savulescu's claim. They speculate that these patients "do not possess self-consciousness, or a desire to go on living, and little to no psychological connectedness over time," (Kahane and Savulescu 2009) and for this reason, they would not be harmed if their lives were to end earlier than they otherwise might. And, in light of this, "considerations of distributive justice" may justify the removal of life-sustaining treatment (Kahane and Savulescu 2009).

Kahane and Savulescu are here referring to patients with minimal sapience. The idea seems to be that even if these patients can experience basic sensory pleasures, and benefit from this experience, their lack of sapience mitigates the prudential value of these experiential interests. The prudential benefit these pleasures provide to the patient does not offset their cost. This is a claim not about a certain conception of well-being (i.e. whether their lives are on balance more pleasurable than painful), but a different claim about the worthwhileness of a life, and the just allocation of health-care resources. Still, it may strike many as compelling. Even if an individual's experience were, on balance, pleasurable, is this kind of existence enough to justify keeping this individual alive, especially if doing so comes at great expense?

In support of this claim, Kahane and Savulescu draw on Jeff McMahan's notion of 'timerelative interests' (McMahan 2002). Briefly, McMahan argues that the degree of rational egoistic concern one has about one's own future varies with the degree of 'psychological unity' that exists between oneself now, and oneself in the future (McMahan 2002). Psychological unity depends on the connectedness and continuity of an individual's psychological makeup over time. The degree of psychological unity within a life is a function of the "proportion of the mental life that is sustained over that period, the richness of that mental life, and the degree of internal reference among the various earlier and later mental states" (McMahan 2002).

An individual's time-relative interests, on the other hand, are what an individual has egoistic reason to care about now (or what a third party has reason to care about for the individual's 
own sake). The strength of these interests is affected by the degree of psychological unity which exists between the individual in the present, and the individual in some future state. If there is a high degree of psychological unity between Joe in the present, and Joe* at some future time, then it is rational for Joe to have a strong time-relative interest in what happens to Joe*, because it makes sense for Joe to think of Joe* as a future state of himself. Conversely, if there is only a minimal degree of psychological unity between Joe and Joe*, it would be rational for Joe to have only a weak time-relative interest in what happens to Joe*; Joe* is, in a manner of speaking, a different person than Joe.

Kahane and Savulescu claim that while patients diagnosed as vegetative with covert awareness may have pleasurable experiences, they stand to lose very little by having their lives ended. Each experience is a discrete, isolated event, with little or no connection to the experiences that precede or follow it. The absence of any persisting structure to the lives of these patients, in anything but a merely biological sense, suggests that even if these patients were to experience pleasurable states in the future, they are not related to their future selves in the sort of way that would allow these pleasurable experiences to contribute to their well-being.

If patients diagnosed as vegetative with covert awareness are akin to 'contented infants', this argument has some force. While their lives may be, on balance, pleasurable, this may not be enough to justify sustaining their lives at great cost. The answer to this question will depend on our societal values - to what extent do we value sustaining human life, despite the cost, and to what extent do we value the most efficient use of health care resources? I will not attempt to answer this question here. In any case, there is substantial evidence to suggest that patients diagnosed as vegetative with covert awareness do possess a degree of 'psychological unity' such that it would matter to them now what happened to their future selves. For example, some patients have used the mental imagery task to communicate with researchers through yes-or-no questions, accurately answering questions about their location in space and time (i.e., the correct date, their current location), and the names of family members. One patient was able to correctly name his personal support worker, whom he had only met after his accident, which suggests that this patient was able to form new memories (Fernandez-Espejo and Owen 2013). This evidence supports the idea that these patients may retain a degree of psychological unity, such that the experience of pleasure and pain accrues to 'the same person', and thus, contributes or detracts from the individual's well-being.

Clearly, at least some patients diagnosed as vegetative with covert awareness possess a degree of psychological unity. They are likely not existing merely 'in the moment,' and thus, may experience significant harm if their lives are ended earlier than they otherwise would be. They are not the kinds of patients that Kahane and Savulescu refer to as possessing only minimal sapience, and thus, an argument that the prudential value of their experiential interests is mitigated by a lack of sapience does not apply to them. Of course, it is an open question whether the experiences of patients diagnosed as vegetative with covert awareness are on balance painful, or pleasurable. On the one hand, the only patient to have been asked directly using the mental imagery task answered 'no' to the question 'are you in any pain?' (FernandezEspejo and Owen 2013). This same patient also indicated that he enjoyed watching hockey on television (Fernandez-Espejo and Owen 2013).

However, at this point, it is less clear that we are talking about the kinds of individuals who could be faring well given the life of a 'contented infant.' Simply having their basic experiential needs met may no longer be enough for individuals who retain cognitive capacities like executive function, to be 'faring well'. Thus, a strictly hedonist account of well-being would not capture all of the constituents of well-being for these individuals. 
Consideration of the experiential interests of patients diagnosed as vegetative with covert awareness does not give us a definitive answer regarding their well-being. On the one hand, if their cognitive capacities are such that they are only capable of experiencing states of pleasure or pain (i.e., if they possess minimal sapience), individuals diagnosed as vegetative with covert awareness may be capable of faring moderately well, provided their basic needs are met. On the other hand, those individuals who retain more sophisticated cognitive capacities may not be faring well with only their experiential interests satisfied. However, in either case, consideration of their experiential interests does not support the claim that patients diagnosed as vegetative with covert awareness would be better off having life-sustaining treatment withdrawn.

\section{Desiderative Interests}

Since a hedonist account of well-being leaves out some important welfare ingredients for (some) individuals diagnosed as vegetative with covert awareness, we must incorporate other kinds of prudential value into our conception of their well-being. Another way in which our lives might go better (or worse), for us, is if our desires are on balance, satisfied (or frustrated). While this conception of well-being has received a great deal of support in the philosophical literature, applying it to these sorts of individuals has its challenges. First, it is not clear what kinds of desires individuals like this can have, nor is it clear what desires they do have. For instance, Kahane and Savulescu suggest that patients diagnosed as vegetative with covert awareness "have, at best, only the most rudimentary desires" (Kahane and Savulescu 2009) and that any desiderative interests they do have would not be generated by present desires, but rather, would be generated by past desires.

In the above passage, Kahane and Savulescu are referring to patients with only minimal sapience. As I suggested previously, however, many patients diagnosed as vegetative likely retain more than minimal sapience. Indeed, at least some patients diagnosed as vegetative with covert awareness can form and express preferences (Fernandez-Espejo and Owen 2013), which suggests that they may also be capable of present desires. Evidence from neuroimaging studies, as well as anecdotal evidence from the family members of patients, is highly suggestive of the fact that some patients diagnosed as vegetative with covert awareness do have desires and preferences about present and future states of affairs. Thus, if patients are capable of communication through neuroimaging, inquiring into their desires could be an important way of investigating and promoting their well-being. While current techniques limit patients to binary communication (i.e., yes-or-no answers), future methods may allow patients to select one of several potential options (i.e., by selectively attending to a particular number signifying one of several options, and not attending to others). Moreover, technology like electroencephalogram (EEG) for brain-based communication is currently being researched (Cruse et al. 2011), and would provide a much more portable and cost-effective option than fMRI, and in turn make regular communication with patients much more practical, and widely accessible. This would allow patients to convey greater detail about their desires and preferences, and allow for a much wider range of communication.

Although we can only speculate at present about the kinds of desires these patients might have, it seems reasonable to assume that many of their desires would be frustrated by their condition. Indeed, while the extent of their cognitive limitations may circumscribe the kinds of desires these patients can have (e.g., they may be incapable of forming abstract desires, such as the desire to achieve meaningful goals), their physical limitations would likely prevent the satisfaction of many basic desires (e.g. a desire to feed oneself, or move around on one's own). 
Still, it is not implausible to think that if these patients could express their present desires, enough of them might be satisfied to yield a passable level of well-being. For example, patients could express their preferences for what kind of entertainment they are provided (e.g., television, movies, music), whether they are satisfied with their care schedule (e.g., feeding, bathing, etc.), and other matters of day-to-day living. ${ }^{2}$ While the satisfaction of these desires may convey only a modest amount of prudential value, given the limited nature of the desires these patients can likely have, it may be enough to partially offset the frustration of other present desires, at least to the extent that they would not be better off having treatment withdrawn.

On the other hand, some patients diagnosed as vegetative with covert awareness may not retain a degree of sapience sufficient for complex desires, while still retaining a degree of sapience sufficient to support psychological connectedness. It is unclear if these patients possess more than 'rudimentary desires', and if so, what these desires might be, (and whether they can be satisfied in the patient's current state). For patients who possess the capacity for only the most rudimentary desires, (i.e., minimal sapience), as well as those whose capacity for desires are unknown (i.e., greater than minimal, but less than 'significant' sapience), we might appeal to their past desires, to determine their desiderative interests in the present. To this end, Kahane and Savulescu focus on a desire whose satisfaction or frustration would likely have a major impact on the overall well-being of these patients. They claim that because most healthy individuals would not desire to be given life-sustaining treatment if they were in a vegetative state — and complete advance directives to this effect - patients currently living in a vegetative state have this putative desiderative interest frustrated by their continued existence. The frustration of this desire is enough to render them better off having life-sustaining treatment withdrawn (even if they could satisfy some present desires). However, it does not follow that the satisfaction of this past desire is now in their best interests, for two reasons. First, this past desire may be misinformed, and thus, its satisfaction may not be consistent with their well-being. Second, the satisfaction of a past desire may fail to be beneficial in the present, if it is no longer endorsed.

\subsection{Misinformed Desires}

On most desire-satisfaction accounts of well-being, the satisfaction of our desires only has prudential value if the desire is properly informed; what intrinsically contributes to my wellbeing is getting what I would want, were I aware of all the non-evaluative facts.

There are reasons for thinking that a past desire not to continue living in a vegetative state with covert awareness might not be sufficiently informed (and thus, that its frustration does not compromise an individual's well-being). Several studies have demonstrated that healthy individuals often misrepresent the degree to which a particular ailment will affect their quality of life, and that this may lead to an unduly pessimistic evaluation of the level of well-being of patients with disease or disability (Amundson 2010). In fact, patients with severe disability or illness tend to systematically evaluate their own lives much more favorably than healthy individuals, while healthy individuals tend to inaccurately predict the degree to which disability or chronic illness will negatively affect their lives (Schkade and Kahnemann 1998; Ubel et al. 2001; Ubel et al.

\footnotetext{
${ }^{2}$ It is worth mentioning that the range of patient desires which can be satisfied may be highly dependant on the circumstances of their care. Individuals living at home may have caregivers who can provide much more individualized care than is available in long-term care facilities. Thus, it may be the case that patients living in long-term care facilities will have a lower level of well-being not as a result of their condition per se, but because of the inability of others to satisfy their desires.
} 
2005). This phenomenon has been referred to as the 'disability paradox' (Albrecht and Devlieger 1999).

Research suggests that our subjective well-being is mediated by our psychological reactions (Amundson 2010). One of the ways in which our psychological reactions influence our judgments of our subjective well-being is through the process of 'hedonic adaptation'; our level of happiness returns towards homeostasis after some life experience causes it to become much higher or lower than it had previously been. For example, individuals with quadriplegia, after a period of adjustment, report levels of well-being similar to persons without disabilities (Albrecht and Devlieger 1999). Conversely, when estimating the level of well-being of others, (or about ourselves in the future), we tend to ignore the mediating effect of psychological factors, and assume that our happiness is a direct function of our circumstances (Amundson 2010). Thus, when considering the wellbeing of an individual who is paraplegic, for example, we imagine that the low level of well-being they experience initially will remain constant for as long as they remain paraplegic, rather than levelling off over time. If healthy people do not take this adaptation process into account, it is likely that they will overestimate the degree to which a disability or illness will compromise their happiness or well-being (Ubel et al. 2005).

Similarly, the 'focussing illusion' has been shown to significantly influence our estimation of well-being in others (Schkade and Kahnemann 1998; Gilbert et al. 1998). According to this theory, subjects' judgements of well-being (either their own, or another person's), can be affected by focussing their attention on positive or negative aspects of the life of the individual being judged. Research measuring the estimated quality of life of individuals with paraplegia has shown that people personally acquainted with someone with paraplegia estimate a much higher level of well-being in these patients (consistent with the reported well-being of these individuals themselves) than those that do not (Schkade and Kahnemann 1998). Rather than focussing only on the injury, those acquainted with people with paraplegia have a more complete perspective of what that individual's life is like.

Given the unfamiliarity of many healthy individuals with what it is like to live with a severe physical or cognitive disability, as well as our general unfamiliarity with what it is like to be in a vegetative state with covert awareness, it is possible that we may overestimate the degree to which being in a vegetative state with covert awareness will negatively impact our lives. Specifically, it may fail to be the case that living in a vegetative state with covert awareness would be worse than having life-sustaining treatment withdrawn. If the desire not to continue living in a vegetative state is based on a certain set of expectations about what this state is like, the fact that our expectations of what life with a significant disability is like - as well as how we will respond to such a life - may be mistaken, suggests that the same might hold true of living in a vegetative state with covert awareness. Therefore, satisfying a past desire not to live in a vegetative state may not be in the best interests of these individuals now.

One might object, however, that empirical research into subjective well-being does not support the claim that we might be mistaken about the well-being of individuals with severe disability. Rather, what this research actually shows is that individuals' self-reports of their own well-being is not a reliable indicator of how they are actually faring. Indeed, people can be happy with their lives — or at least, think they are - in situations where we would not want to say that they are faring well. Thus, if individuals with severe disabilities report a high level of well-being, we might think, they must either be mistaken about their own well-being, or the measures of well-being used to generate these results must be flawed. And, if individuals with severe disability are mistaken about their own well-being (i.e., they really are faring poorly), 
then the past desire of a healthy individual not to continue living in a vegetative state (because such an existence is intolerably bad) may not be misinformed.

Nevertheless, even if there is legitimate concern that reports of subjective well-being or happiness can be mistaken, there is still good reason to think that subjective well-being or happiness is an important part of well-being. Even if one's happiness is not equivalent to their well-being, surely an individual's happiness contributes to their well-being in a positive way. Thus, if a patient diagnosed as vegetative with covert awareness claimed to be happy, it may still be the case that they are faring poorly, but it seems much less likely that they are faring so poorly that they would be better off having life-sustaining treatment withdrawn. Thus, insofar as a past desire not to continue living in a vegetative state is based on the belief that such a state is 'worse than death', this desire might be misinformed.

\subsection{Desires No Longer Endorsed}

Even if a desire for $\mathrm{X}$ was well-informed, it may be the case that when the time comes for the desire to be satisfied, the individual may no longer desire $\mathrm{X}$. In the case of patients diagnosed as vegetative with covert awareness, it may be the case that these patients had a past desire not to continue living in a vegetative state, but now that they are in a vegetative state, they no longer hold that desire. In what sense, then, would satisfying that desire promote their well-being?

We can see a concrete example of the potential for an individual's past desires to inaccurately reflect their current well-being by considering a patient population which in many ways resembles the patient population under consideration: patients with locked-in syndrome. Locked-in patients are incapable of voluntary movement (except, in most cases, for vertical eye movement) or verbal communication, although they remain fully aware. Surprisingly, many of these patients report a positive quality of life (Laureys et al. 2005). A recent study by Bruno and colleagues surveyed a group of 65 patients with locked-in syndrome, $72 \%$ of whom indicated that they were happy, with a greater period in the locked-in state positively correlated with patient happiness (Bruno et al. 2011). Other studies have found that patient-evaluated quality of life was not significantly different from healthy controls (with the exception being on scales of physical function) (Lule et al. 2009; Rousseau et al. 2013). The fact that these patients report that they are happy in their state suggests that it may not be in their best interests to withdraw them from life sustaining treatment. While there are significant differences between patients with locked-in syndrome, and patients diagnosed as vegetative with covert awareness (e.g. ability to communicate, cognitive capacities), the example of locked-in patients illustrates how an individual might view a potential state of affairs with disapprobation, and yet, when in this state, view it much more favorably.

In fact, some patients diagnosed as vegetative with covert awareness may more closely resemble those in a locked-in state, than they do typical vegetative state patients. Like lockedin patients, they retain a degree of sapience, while lacking the ability to respond behaviourally to stimuli. In both patient groups, the means of satisfying one's experiential and desiderative interests is severely curtailed. Yet, many locked-in patients report a reasonably high level of life-satisfaction. Insofar as we think that an individual's assessment of their own well-being incorporates their experiential and desiderative interests, this suggests that patients in the locked-in state continue to satisfy at least a passable amount of their experiential and desiderative interests. Thus, the example of locked-in patients presents a counterexample to Kahane and Savulescu's assertion that the desiderative and experiential interests of the lockedin patient (and by extension, the patient diagnosed as vegetative with covert awareness who 
retains significant sapience) are frustrated, and as a result, that such a patient would be better off not continuing to live.

Still, one might raise the following objection: Part of what we are talking about when we talk about the prudential value of a life is how the life fits together as a whole. Thus, how good my life is for me is determined not only by summing the value of the various parts of my life, but also the value of the whole, a value not associated or contained within any single part. We might call this the 'thematic unity' or 'narrative value' of a life (Dworkin 1994; McMahan 2002). In judging what is most consistent with the well-being of an individual diagnosed as vegetative with covert awareness, we must consider the character of her life as a whole, including who she was and what she desired in the past. Living in a vegetative state may be so deeply disruptive to the character of one's life as a whole, that it would be best for that individual not to go on living in such a state. This is one way of understanding Kahane and Savulescu's remark that "remaining conscious...yet losing all the aspects of sapience that constituted you as a person with particular projects and attachments, is more meaningless and degrading than to lose consciousness completely" (Kahane and Savulescu 2009). The past desires of these individuals - including the desire not to go on living in a vegetative state - shaped their lives in a certain way, and determine the sort of life ending which would be best for them. Considering their lives as a whole, it would be best for them to be withdrawn from life-sustaining treatment.

But why should we think that the past desires of an individual override their current interests in determining what is good for them? As Jennifer Hawkins (2014) points out, most theories of welfare either incorporate or are consistent with what she calls a 'nonalienness principle' (NA). NA states that a necessary condition for X's being intrinsically good for person A, is that A responds positively to $X$ at time $T_{1}$ if she is aware of it at $T_{1}$, or $A$ would respond positively to $X$ at $T_{1}$, if she were aware of $X$ at $T_{1}$. Accepting this principle entails that satisfying a past desire cannot be good for an individual, unless the individual experiences the satisfaction of that desire as positively valenced. This does not mean that narrative or thematic goods are impossible; rather, thematic unity is prudentially valuable only insofar as we continue to experience it in a positively valenced way, or would, if we were aware of it. In the case of patients diagnosed as vegetative with covert awareness, the satisfaction of a past desire not to continue living in such a state is only prudentially valuable insofar as it remains consistent with the current values and concerns of the individual. If these individuals are only minimally sapient and lack psychological connectedness over time - if the narrative value of their lives is no longer positively valanced for them - it is false that satisfying this past desire is a benefit to them.

Conversely, if these individuals retain significant sapience, and do retain psychological connectedness - as I have suggested - the satisfaction of the past desire not to continue living may be in their interest now. Yet, as I alluded to above, there is some reason for thinking this may not be the case. (It is here that the example of locked-in patients is potentially illuminating). What is good for me must depend in some respect on what I am like. As I change (i.e., as my attitudes, values, dispositions, etc. change), what is good for me changes as well. This is where individuals diagnosed as vegetative with covert awareness present an interesting case. A catastrophic brain injury, resulting in significant physical and cognitive impairments, would seem to be the kind of life-event which would result in a major shift in one's values, attitudes, and disposition. Conversely, it may not even be within the conceptual abilities of these individuals to desire not to continue existing. This is not to say that we should not infer some of the desires of these individuals from what we knew about them in the past. Previously held desires may be good predictors of current interests; this seems like a good place to start when thinking of day-to-day actions which might promote the welfare of these individuals. However, it is not clear that a past desire not to continue 
living in a vegetative state is a good predictor of an equivalent current desire, and thus, that satisfying such a desire would benefit the individual.

\section{Objective Interests}

The discussion of the last two sections has shown that consideration of the experiential and desiderative interests of individuals diagnosed as vegetative with covert awareness does not give a clear indication of their welfare. This is in part because it is not clear that these views encapsulate what it means for these individuals to fare well, and in part because of the lack of insight we have into the experiences and cognitive capacities of these individuals. To avoid this latter issue, then, we might consider a view of well-being that does not (necessarily) rely on the subjective attitudes, desires or mental states of the individual. Objective list views hold that what is non-instrumentally good for a person is the possession of objectively valuable goods, and what is non-instrumentally bad for a person is the possession of objectively disvaluable evils (or a lack of objectively valuable goods). To fare well is to possess more objective goods than objective evils.

Kahane and Savulescu suggest that because patients diagnosed as vegetative with covert awareness remain conscious, and yet, have lost the cognitive and motivational capacities which constitute them as a person, we might think that continued existence is "more meaningless and degrading than to lose consciousness completely" (Kahane and Savulescu 2009). The idea is that these individuals lack virtually all of the sorts of goods which constitute wellbeing. They cannot cultivate deep personal relationships, pursue their projects, develop their talents, or satisfy other objective interests. The lack of these objective goods from their lives, then, justifies the intuition that these patients are faring poorly. Undoubtedly, there are aspects of normal human life which will no longer be accessible to patients diagnosed as vegetative with covert awareness. But does it follow that these individuals are faring so poorly that they would be better off no longer existing?

On the one hand, when we consider the well-being of these individuals, we typically do so from the perspective of the entirety of their lives. We can envision how their lives might have been otherwise, and how their injury has deprived them of a great deal of good. It is this loss of future prudential good which compromises their well-being now. However, it is not clear that these individuals experience things in this way. They may lack the cognitive capacities to contextualize their current state, or to appreciate the objective goods which are now unavailable to them. In a sense, the objective goods of a healthy, adult life may no longer exist for them. They do not experience the inability to develop their talents, pursue their former projects, or cultivate knowledge as a harm, or a loss, because they are no longer aware of these goods. And, insofar as they can no longer conceptualize these goods, it follows that they can no longer value them.

Does the fact that these patients have lost something they no longer value compromise their well-being? How one answers this question will depend on how strongly one favors objective theories of well-being. For example, for most paradigmatic objective goods, we can imagine an individual who simply does not value the possession of that good. Some people simply do not value achievement, or relationships with others, or cultivating their talents. If this is genuinely the case (i.e., these individuals are not mistaken about what they actually value, or would value given sufficient exposure), are these individuals really worse off for lacking these goods? This is a real tension between objective and subjective theories of well-being in 
general; specifically, that objective accounts seem to permit that prudential goods may be inconsistent with (or 'alien') to an individual's sincerely held values and attitudes.

However, whereas we may not think that the hypothetical individuals above would be better off having certain objective goods, it certainly seems as though patients diagnosed as vegetative with covert awareness would be, which suggests that their well-being is compromised by the lack of these goods. Still, even if we grant that on an objective view of wellbeing, these patients have suffered a tremendous loss of prudential value, the fact that they do not experience this loss directly does seem to mitigate their ill-being somewhat. Intuitively, this seems to be better than the case in which the patient is cognizant of the objective goods they have lost. Provided they can experience other prudential goods (i.e., experiential goods) to a sufficient degree, they would seem to be better off continuing to exist, even if their level of well-being is far lower than where it could have been otherwise.

Conversely, some patients diagnosed as vegetative with covert awareness may remain cognizant of, and continue to value, goods like pursuing their goals, cultivating their talents, and maintaining personal relationships. Surely these patients, we might think (and Kahane and Savulescu assert), are faring very poorly; having deep personal relationships, pursuing their goals and projects, etc. would be good for them, and the fact that these interests are universally frustrated makes their existence 'a fate worse than death'.

I agree with Kahane and Savulescu that the well-being of these patients has been severely compromised by their injury. Nevertheless, I think that their conclusion is overly pessimistic, and one that is not clearly borne out by the reports of similarly situated individuals. As I mentioned above, many locked-in patients report that they are more-or-less happy in their current state, and would not want to have life-sustaining treatment withdrawn. Granted, many locked-in patients can communicate in some way, which may in turn expand the range of objective goods that they can pursue. Nevertheless, it seems at least plausible that 'higher-functioning' patients diagnosed as vegetative with covert awareness of the sort that we would be most concerned about might similarly adjust their values and expectations in light of their injuries.

For example, Petry et al. (2009) have developed a quality of life assessment designed for individuals with profound multiple disabilities (i.e., profound intellectual disabilities, in addition to motor and sensory limitations), which includes common domains of well-being like physical well-being, emotional well-being, interpersonal relations, material well-being, self-determination, personal development, and social inclusion. While these domains track those constituents of well-being typically cited by objective-list theories, the ingredients of well-being for these individuals are much more modest. Thus, for an individual diagnosed as vegetative with covert awareness, social-inclusion may be partially realized by regular visits from family, while relatively simple choices (e.g., choosing what kind of music to listen to), may contribute to self-determination.

Undoubtedly, the level of well-being of individuals with such extensive cognitive and motor limitations is far below what we would want for ourselves or those we care about. The maximum level of well-being which might be achieved by patients diagnosed as vegetative with covert awareness - at least on an objective account of well-being - is probably quite low. Moreover, the extent to which they might realize certain objective goods would likely make only a modest contribution to their overall well-being. Yet these gains aren't inconsequential. Perhaps they would be enough to reach a level of well-being that, though much lower than it might otherwise have been, would not be so low that these patients would be better off not existing. Researchers have not yet asked patients diagnosed as vegetative with covert awareness questions about their well-being; I think that the reports of locked-in patients are 
suggestive of what their responses would be like. People can be remarkable resilient in the face of tremendous adversity, and it seems plausible that patients diagnosed as vegetative with covert awareness may be similarly capable of recalibrating their expectations and values, such that even a severely diminished existence may not be "more meaningless and degrading than to lose consciousness completely" (Kahane and Savulescu 2009). Given this possibility, it would be rash to conclude that such patients would be better off not existing.

Moreover, there are practical reasons to resist Kahane and Savulescu's pessimistic conclusion. First, the objective goods of these individuals may be frustrated simply because of a lack of care. This is perhaps the most dangerous part of adopting a pessimistic perspective like that of Kahane and Savulescu about the prospects of these individuals' well-being. If we adopt the attitude that these individuals are incapable of realizing prudential goods, we might think 'what is the point of trying to promote their well-being'? This may result in many of the individuals being left to languish in long-term care facilities, with little stimulation or human contact. For example, Puggina and colleagues describe how even when cognizant of the fact that some patients may be covertly aware, nurses in intensive care facilities often find it challenging to maintain verbal interaction, as well as pain and symptom management, with patients. Research has indicated that $90 \%$ of care procedures are done in silence, with other studies suggesting that nurses may spend less than 5\% of their time verbally communicating with patients (Puggina et al. 2012). Simple actions like talking to the patient, guiding them through care procedures, identifying them by their own name, and avoiding parallel conversations, demonstrates respect, and recognition of the fact that they are still persons.

It is worth noting that Kahane and Savulescu argue that, insofar as patients diagnosed as vegetative with covert awareness have experiential interests, these interests create a moral reason to alleviate pain as well as make these patients as comfortable as possible for as long as they are alive. This is surely correct. However, I suggest, promoting the well-being of these patients goes beyond maintaining physical comfort; it requires that we treat these patients with respect, dignity, and concern for their interests and values.

Second, continued research efforts into communicating with these individuals, both through functional neuroimaging and other means (e.g., EEG), may yield further means of enriching their lives. Given that some individuals diagnosed as vegetative with covert awareness have shown evidence of similar conscious experiences as healthy individuals when provided with naturalistic stimuli, such as movies (Naci et al. 2013; Naci et al. 2017) or jokes (Fiacconi and Owen 2016), it seems likely that the ingredients of their well-being go beyond the satisfaction of basic needs. These individuals likely have present desires, and perhaps even desires about the future. Communicating with these individuals could provide invaluable insight into their well-being, and ways of promoting their interests. Yet, the impetus for this research is threatened if we are prepared to simply assume that these individuals are incapable of possessing the sorts of objective goods (or other prudential goods) that contribute to well-being.

\section{Conclusion}

Determining the well-being of individuals diagnosed as vegetative with covert awareness is challenging. On the one hand, they represent a heterogenous population, whose residual cognitive capacities may differ significantly. On the other hand, their ability to communicate is severely limited, making it difficult (if not impossible) to discern first-hand what their prudential interests are, and whether they are satisfied. This has led others to speculate that these individuals are faring so poorly that they would be better off not existing. I think that we 
should resist this conclusion. Consideration of the experiential and desiderative interests does not clearly support the conclusion that they are faring poorly. And, while an objective account of well-being would hold that the well-being of (some of) these patients is severely compromised, it is not clear that these patients would be better off not existing.

I think we should resist the temptation to evaluate the well-being of these individuals in the same way that we would healthy, adult humans. Evaluating the well-being of these individuals is not as simple as imagining the life of a healthy individual, and subtracting those prudential goods which are frustrated by their diminished physical and cognitive state. Not only may their capacity to appreciate certain prudential goods have changed, but the sorts of prudential goods that they value may have changed as well. When thinking about the well-being of individuals diagnosed as vegetative with covert awareness, we must account for the potential for a recalibration of what these individuals consider valuable or good. We need to be able to adopt the perspective of these individuals. As I have shown, the account given by Kahane and Savulescu fails to do this.

The purpose of this paper is not to argue that these patients are faring well, but to put forward the idea that with the attentive care of family members, and members of the health-care team, it is possible that these individuals might have lives of at least decent quality. Even if this is not the kind of life we would desire for ourselves or those we care about, it does not follow that the lives of these individuals are intolerably poor. I have tried to avoid speculating about what the well-being of these individuals actually is, while trying to provide an alternative perspective to the negative assessment exemplified by Kahane and Savulescu. More research is needed, involving these individuals directly, to better understand their experience, and the extent to which their prudential interests are satisfied or frustrated. Before advocating for the withdrawal of life-sustaining treatment, this seems like the least we can do for them.

Acknowledgements Dr. Charles Weijer, and Dr. Anthony Skelton, for many helpful comments on earlier drafts of this paper, as well as two anonymous reviewers for this journal.

Open Access This article is distributed under the terms of the Creative Commons Attribution 4.0 International License (http://creativecommons.org/licenses/by/4.0/), which permits unrestricted use, distribution, and reproduction in any medium, provided you give appropriate credit to the original author(s) and the source, provide a link to the Creative Commons license, and indicate if changes were made.

\section{References}

Albrecht GL, Devlieger PJ (1999) The disability paradox: high quality of life against all odds. Soc Sci Med 48(8):977-988

Amundson R (2010) Quality of life, disability, and hedonic psychology. J Theory Soc Behav 40(4):374-392

Bardin JC, Schiff ND, Voss HU (2012) Pattern classification of volitional functional magnetic resonance imaging responses in patients with severe brain injury. Arch Neurol 69:176-181

Bruno MA, Bernheim JL, Ledoux D, Pellas F, Demertzi A, Laureys S (2011) A survey on self-assessed wellbeing in a cohort of chronic locked-in syndrome patients: happy majority, miserable minority. BMJ Open 1(1):e000039

Coleman MR, Davis MH, Rodd JM, Robson T, Ali A, Owen AM, Pickard JD (2009) Towards the routine use of brain imaging to aid the clinical diagnosis of disorders of consciousness. Brain 132(9):2541-2552

Cruse D, Chennu S, Chatelle C, Bekinschtein T, Fernandez-Espejo D, Pickard JD, Laureys S, Owen AM (2011) Beside detection of awareness in the vegetative state: a cohort study. Lancet 378(9809):2088-2094

Dworkin R (1994) Life's dominion. Vintage Books, New York

Fernandez-Espejo D, Owen AM (2013) Detecting awareness after severe brain injury. Nat Rev Neurosci 14(11): 801-809 
Fiacconi C, Owen AM (2016) Using facial electromyography to detect preserved emotional processing in disorders of consciousness: a proof-of-principle study. Clin Neurophysiol 127:3000-3006

Gilbert DT, Pinel EC, Wilson TD, Blumberg SJ, Wheatley T (1998) Immune neglect: a source of durability bias in affective forecasting. J Pers Soc Psychol 75:617-638

Gosseries O, Zasler ND, Laureys S (2014) Recent advances in disorders of consciousness: focus on the diagnosis. Brain Inj 28(9):1141-1150

Graham M, Weijer C, Cruse D, Fernandez-Espjeo D, Gofton T, Gonzalez-Lara L et al (2015) An ethics of welfare for patients diagnosed as vegetative with covert awareness. AJOB Neurosci 6(2):31-41

Hawkins J (2014) Well-being, time, and dementia. Ethics 124(3):507-542

Kahane G, Savulescu J (2009) Brain damage and the moral significance of consciousness. J Med Philos 34(1):6-26

Laureys S, Schiff ND (2012) Coma and consciousness: paradigms (re)framed by neuroimaging. NeuroImage 61(2):478-491

Laureys S, Pellas F, Van Eeckhout P, Ghorbel S, Schnakers C, Perrin F et al (2005) The locked-in syndrome: what is it like to be conscious but paralyzed and voiceless? Prog Brain Res 150:495-511

Lule D, Zickler C, Hacker S, Bruno MA, Demertzi A, Pellas F et al (2009) Life can be worth living in locked-in syndrome. Prog Brain Res 177:339-351

McMahan J (2002) The ethics of killing: problems at the margins of life. Oxford University Press, Oxford

Monti MM, Vanhaudenhuyse A, Coleman MR, Boly M, Pickard JD, Tshibanda L et al (2010) Willful modulation of brain activity in disorders of consciousness. N Engl J Med 362(7):579-589

Naci L, Cusack R, Jia VZ, Owen AM (2013) The brain's silent messenger: using selective attention to decode human thought for brain-based communication. J Neurosci 33(22):9385-9393

Naci L, Cusack R, Anello M, Owen AM (2014) A common neural code for similar conscious experiences in behaviourally non-responsive patients. PNAS 111:14277-14282

Naci L, Graham M, Weijer C, Owen AM (2017) Covert narrative capacity: mental life in patients thought to lack consciousness. Ann Clin Transl Neurol 4(1):61-70

Owen AM, Coleman MR, Boly M, Davis MH, Laureys S, Pickard JD (2006) Detecting awareness in the vegetative state. Science 313(5792): 1402

Peterson A, Cruse D, Naci L, Weijer C, Owen AM (2015) Risk, diagnostic error, and the clinical science of consciousness. NeuroImage: Clin 7:588-597

Petry K, Maes B, Vlaskamp C (2009) Measuring the quality of life of people with profound multiple disabilities using the QOL-PMD: first results. Res Dev Disabil 30(6):1394-1405

Puggina ACG, da Silva MJP, Schnakers C, Laureys S (2012) Nursing care of patients with disorders of consciousness. J Neurosci Nurs 44(5):260-270

Rousseau MC, Pietra S, Nadji M, Billette de Villemeur T (2013) Evaluation of quality of life in complete lockedin syndrome patients. J Palliat Med 16(11):1455-1458

Royal College of Physicians (2003) The vegetative state: guidance on diagnosis and management. Clin Med 3(3):249-254

Schkade DA, Kahnemann D (1998) Does living in California make people happy? Psychol Sci 9:340-346

Skelton A (2016) Children's well-being: a philosophical analysis. In: Fletcher G (ed) The Routledge handbook of philosophy of well-being. Routledge, New York, pp 366-377

Stender J, Gosseries O, Bruno MA, Charland-Verville V, Vanhuadenhuyse A, Demertzi A et al (2014) Diagnostic precision of PET imaging and functional MRI in disorders of consciousness: a clinical validation study. Lancet 384(9942):514-522

The Multi-Society Task Force on PVS (1994) Medical aspects of the persistent vegetative state. N Engl J Med 330:1499-1508

Ubel PA, Loewenstein G, Hershey J, Baron J, Mohr T, Asch DA et al (2001) Do non-patients underestimate the quality of life associated with chronic health conditions because of a focusing illusion? Med Decis Mak 21: 190-199

Ubel PA, Loewenstein G, Schwartz N, Smith D (2005) Misimagining the unimaginable: the disability paradox and health care decision making. Health Psychol 24(4):S57-S62 